\title{
Dilated Cardiomyopathy With Mid-Range Ejection Fraction at Diagnosis: Characterization and Natural History
}

Piero Gentile, MD; Marco Merlo, MD; Antonio Cannatà, MD; Marco Gobbo, MD; Jessica Artico, MD; Davide Stolfo, MD; Marta Gigli, MD; Federica Ramani; Giulia Barbati, PhD; Bruno Pinamonti, MD; Gianfranco Sinagra, MD

Background-Limited data are available on mid-range ejection fraction (mrEF) patients with dilated cardiomyopathy. We sought to define the characteristics, evolution, and long-term prognosis of dilated cardiomyopathy patients with mrEF at diagnosis.

Methods and Results-We analyzed all dilated cardiomyopathy patients consecutively evaluated in the Trieste Heart Muscle Disease Registry from 1988 to 2013. mrEF and reduced ejection fraction (rEF) were defined as baseline left ventricular (LV) ejection fraction values between $40 \%$ and $49 \%$ and $<40 \%$, respectively. All-cause mortality or heart transplantation, sudden cardiac death, or major ventricular arrhythmias were considered as outcome measures. Worsening LV ejection fraction (reduction to $<40 \%$ ) during follow-up was also considered to identify possible predictors of adverse remodeling. Among 812 enrolled patients, 175 (22\%) presented with mrEF at presentation. At baseline, as compared with the rEF group, mrEF patients had lower rates of moderate-severe mitral regurgitation and restrictive LV filling pattern. During a median follow-up period of 120 (60-204) months, the mrEF group presented a lower rate of death/heart transplantation ( $9 \%$ versus $36 \%, P<0.001$ ) and sudden cardiac death or major ventricular arrhythmias (4.5\% versus 15\%, $P<0.001)$ than rEF patients. Moreover, 29 out of $175 \mathrm{mrEF}$ patients (17\%) evolved to rEF. Restrictive LV filling pattern emerged as the strongest predictor of rEF development following multivariable analysis.

Conc/usions-mrEF identified a consistent subgroup of dilated cardiomyopathy patients diagnosed in an earlier stage with subsequent apparent better long-term evolution. However, $17 \%$ of these patients evolved into rEF despite the use of medical therapy. A baseline restrictive LV filling pattern was independently associated with subsequent evolution to rEF. ( $\boldsymbol{A}$ Am Heart Assoc. 2019;8:e010705. DOI: 10.1161/JAHA.118.010705.)

Key Words: dilated cardiomyopathy • heart failure • mid-range ejection fraction • prognosis

$\mathrm{T}$ he European Society of Cardiology has introduced a novel class of heart failure (HF) without a severely reduced ejection fraction (EF), calling it as $\mathrm{HF}$ with a midrange EF. HF with a mid-range EF mainly includes patients with primarily mild systolic dysfunction and features of diastolic dysfunction. ${ }^{1}$ This new entity encompasses patients with clinical, laboratory, or instrumental evidence of HF and a left ventricular ejection fraction (LVEF) between $40 \%$ and $49 \%$. The definition of this new class of HF could

From the Cardiothoracic Department, Azienda Sanitaria Universitaria Integrata di Trieste and University of Trieste, Italy (P.G., M.M., A.C., M. Gobbo, J.A., D.S., M. Gigli, F.R., B.P., G.S.); Biostatistics Unit, Department of Medical Sciences, University of Trieste, Italy (G.B.).

Correspondence to: Merlo Marco, MD, Cardiothoracic Department, Azienda Sanitaria Universitaria Integrata di Trieste and University of Trieste, Via P. Valdoni 7, 34100 Trieste, Italy. E-mail: marco.merlo79@gmail.com

Received December 1, 2018; accepted June 26, 2019.

(C) 2019 The Authors. Published on behalf of the American Heart Association, Inc., by Wiley. This is an open access article under the terms of the Creative Commons Attribution-NonCommercial License, which permits use, distribution and reproduction in any medium, provided the original work is properly cited and is not used for commercial purposes. lead to a better understanding of the characteristics, pathophysiology, and treatment options for a group of patients often neglected in randomized clinical trials. ${ }^{1}$ Dilated cardiomyopathy (DCM), a heterogeneous myocardial disease with a frequent genetic cause, is characterized by several degrees of reduced LVEF. ${ }^{2-4}$ Notably, DCM patients are typically relatively young, with low comorbidities and long periods of asymptomatic LV systolic dysfunction. ${ }^{2}$ They are often diagnosed in an early phase of the disease, through the application of extensive genetic counseling programs and sometimes by way of the results of preparticipation screenings for sport activities. ${ }^{5,6}$ Therefore, at diagnosis, DCM patients could be characterized by the absence of HF symptoms and non-severe systolic dysfunction. However, few data on the characteristics, evolution, and outcomes of DCM patients presenting with mid-range ejection fraction (mrEF) at baseline are available at this time. ${ }^{7}$ In the present study, we sought to characterize DCM patients presenting with mrEF at baseline, describing their natural history and prognosis in comparison with reduced EF (rEF) patients. 


\section{Clinical Perspective}

\section{What Is New?}

- This article includes the largest-to-date patient population with dilated cardiomyopathy and mid-range ejection fraction at first presentation.

- Additionally, it provides the first long-term outcome in these patients with a median follow-up period of 120 (60-204) months.

\section{What Are the Clinical Implications?}

- Patients with mid-range ejection fraction denotes a consistent (ie, 22\%) subgroup of dilated cardiomyopathy deserving careful and reliable risk assessment.

- A non-negligible number of patients (17\%) with mid-range ejection fraction at baseline tend to evolve towards worse clinical phenotype, with a subsequent worse prognosis.

- The presence of a restrictive left ventricular filling pattern at baseline might identify those mid-range ejection fraction patients with a greater risk to experience worse outcomes during follow-up.

\section{Methods}

The authors declare that all supporting data are available within the article.

\section{Study Population}

All DCM patients consecutively enrolled in the Trieste Heart Muscle Disease Registry ${ }^{5}$ from January 1988 to December 2013 were considered eligible for inclusion in the present study and were retrospectively analyzed. Enrolled patients were directly hospitalized in our referral center or referred shortly after the onset of symptoms from other centers.

Follow-up ended on December 31, 2015 or at the time of death or urgent heart transplant (D/HTx).

The institutional ethics board approved the study and written informed consent was obtained under the institutional review board policies of the hospital administration.

A diagnosis of DCM was determined according to the currently accepted criteria. ${ }^{8,9}$ Ischemic etiology was excluded with coronary angiography, systematically performed in patients aged $>35$ years with cardiovascular risk factors. Patients with significant coronary artery disease $>50 \%$ stenosis of a major coronary artery), history of severe systemic hypertension (>160/100 mm Hg), biopsy-proven active myocarditis, alcohol intake of $>100 \mathrm{~g} /$ day, significant organic valve disease, tachycardia-induced cardiomyopathy, peripartum cardiomyopathy, congenital heart disease, or advanced systemic disease affecting their short-term prognosis were excluded. ${ }^{5,10,11}$

After enrollment, if not contraindicated, all patients received evidence-based pharmacological (at maximum tolerated dosages) and device therapies. ${ }^{1}$

Per protocol, all patients underwent a scheduled follow-up (clinical and echocardiographic) at 6, 12, and 24 months after enrollment and then every $\geq 2$ years frequently with respect to individual clinical necessity.

We divided our population into 2 groups according to the baseline EF: patients with rEF (ie, EF <40\%) and patients with mrEF (ie, EF 40\%-49\%).

Table 1. Baseline Clinical Characteristics of the Population Divided According to LVEF Categories

\begin{tabular}{|c|c|c|c|c|}
\hline & $\begin{array}{l}\text { Total } \\
812 \mathrm{pts}\end{array}$ & $\begin{array}{l}\text { rEF DCM } \\
637 \mathrm{pts}\end{array}$ & $\begin{array}{l}\text { mrEF DCM } \\
175 \text { pts }\end{array}$ & $P$ Value \\
\hline Age, y & $46(36 ; 56)$ & $48(37 ; 57)$ & $42(32 ; 50)$ & 0.001 \\
\hline Male sex (\%) & $565(70 \%)$ & $455(71 \%)$ & $110(63 \%)$ & 0.029 \\
\hline NYHA III-IV, n (\%) & $195(24)$ & $186(29)$ & $9(5)$ & $<0.001$ \\
\hline $\mathrm{SBP}, \mathrm{mm} \mathrm{Hg}$ & $124 \pm 18$ & $123 \pm 18$ & $126 \pm 17$ & 0.27 \\
\hline Heart rate, bpm & $78 \pm 16$ & $80 \pm 16$ & $70 \pm 12$ & $<0.001$ \\
\hline LBBB (\%) & $256(32 \%)$ & $221(35 \%)$ & $35(20 \%)$ & $<0.001$ \\
\hline \multicolumn{5}{|l|}{ Echocardiography } \\
\hline LAESAI, $\mathrm{cm}^{2} / \mathrm{m}^{2}$ & $14 \pm 4$ & $14 \pm 4$ & $11 \pm 3$ & $<0.001$ \\
\hline LVEDDI, mm/m² & $36 \pm 6$ & $37 \pm 6$ & $32 \pm 4$ & $<0.001$ \\
\hline LVEDVI, $\mathrm{mL} / \mathrm{m}^{2}$ & $99 \pm 37$ & $106 \pm 37$ & $71 \pm 18$ & $<0.001$ \\
\hline LVEF, \% & $31 \pm 10$ & $27 \pm 7$ & $44 \pm 3$ & $<0.001$ \\
\hline RVD, n (\%) & $199(26)$ & $182(30)$ & $17(10)$ & $<0.001$ \\
\hline $\begin{array}{l}\text { Moderate-severe } \\
\text { MR, } n(\%)\end{array}$ & $285(36)$ & $262(42)$ & $23(13)$ & $<0.001$ \\
\hline $\begin{array}{l}\text { Restrictive LV } \\
\text { filling pattern, } \\
n(\%)\end{array}$ & $233(30)$ & $225(37)$ & $8(5)$ & $<0.001$ \\
\hline $\mathrm{E} / \mathrm{E}^{\prime}$ & $14 \pm 7$ & $15 \pm 8$ & $10 \pm 3$ & $<0.001$ \\
\hline sPAP & $35 \pm 14$ & $37 \pm 15$ & $25 \pm 5$ & $<0.001$ \\
\hline \multicolumn{5}{|l|}{ Therapy } \\
\hline $\begin{array}{c}\text { ACE-inhibitors- } \\
\text { ARBs, n (\%) }\end{array}$ & $783(96)$ & $624(98)$ & $159(91)$ & 0.081 \\
\hline $\begin{array}{l}\text { Beta-blockers, } \\
\text { n (\%) }\end{array}$ & $729(90)$ & $576(91)$ & $153(88)$ & 0.311 \\
\hline Diuretics, n (\%) & $607(75)$ & $531(84)$ & $76(44)$ & $<0.001$ \\
\hline MRAs, n (\%) & $239(30)$ & $216(34)$ & $23(13)$ & $<0.001$ \\
\hline
\end{tabular}

Values are expressed as mean $\pm S D$ or median with interquartile range as appropriate, and as percentage. ACE indicates angiotensin-converting enzyme; ARBs, angiotensin receptor blockers; LAESAI, left atrial end systolic area indexed; LBBB, left bundle branch block; LV, left ventricular; LVEDDI, left ventricular end-diastolic diameter indexed; LVEDVI, left ventricular end-diastolic volume indexed; LVEF, left ventricular ejection fraction; MR, mitral regurgitation; MRAs, mineralocorticoid receptor antagonists; NYHA, New York Heart Association; RVD, right ventricular dysfunction; SBP, systolic blood pressure; sPAP, systolic pulmonary artery pressure. 


\section{Outcome Measures}

The study analyzed the incidence of the following combined outcome measures: (1) all-cause mortality and heart transplantation (D/HTx) and (2) sudden cardiac death (SCD) or major ventricular arrhythmias (MVAs). Specifically, MVAs were defined as sustained $(\geq 30$ seconds, hemodynamically symptomatic) ventricular tachycardia, ventricular fibrillation (VF), appropriate treatment of sustained ventricular tachycardia or ventricular fibrillation via implantable cardioverter-defibrillator therapy (shock or antitachycardia pacing for termination of sustained ventricular tachycardia $\geq 185 \mathrm{bpm}$ ), or cardiopulmonary resuscitation after cardiac arrest. ${ }^{12}$ Finally, a worsening of LVEF to $<40 \%$ during follow-up in the group of patients initially presenting with mrEF was also considered to identify possible predictors of adverse remodeling.

\section{Echocardiographic Study}

Biventricular dimensions and systolic and diastolic functions were assessed according to international guidelines. ${ }^{13}$ Specifically, LV volume and LVEF were calculated by the Simpson biplane method. All volumes were indexed according to body surface area.

Transmitral E- and A-wave velocities were measured using pulsed wave Doppler at the level of the mitral leaflet tips. The LV filling pattern was classified as restrictive in the presence of an E-wave deceleration time of $<120 \mathrm{~ms}$ or $E / A$ value $\geq 2$ associated with an E-wave deceleration time of $\leq 150 \mathrm{~ms}^{.}{ }^{14}$ Right ventricular dysfunction was defined by a right ventricular fractional area change of $<35 \% .{ }^{15}$

Mitral regurgitation (MR) was assessed using a multiparametric approach applied by measuring the effective regurgitant volume or orifice area as determined by the proximal isovelocity surface area, whenever feasible, or the vena contracta width at color-flow Doppler, and graded on a 3-point scale according to current recommendations. ${ }^{16}$ Significant MR was considered if greater than mild. ${ }^{17}$ Left atrial endsystolic area was calculated from a 4-chamber view.

All measurements were obtained from the mean of 3 beats (patients in sinus rhythm) or of 5 beats (patients with atrial fibrillation).

\section{Statistical Analysis}

Summary statistics of clinical and laboratory variables were expressed as means and standard deviations, medians and interquartile ranges, or counts and percentages, as appropriate. Cross-sectional comparisons between groups were made using the ANOVA test on continuous variables, the BrownForsythe statistic when the assumption of equal variances did not hold, or the non-parametric Mann-Whitney test when necessary. The chi-squared or Fisher exact test was used for discrete variables. Repeated measures between baseline and follow-up were evaluated by means of the paired $t$ test for continuous Gaussian-distributed parameters or the Wilcoxon

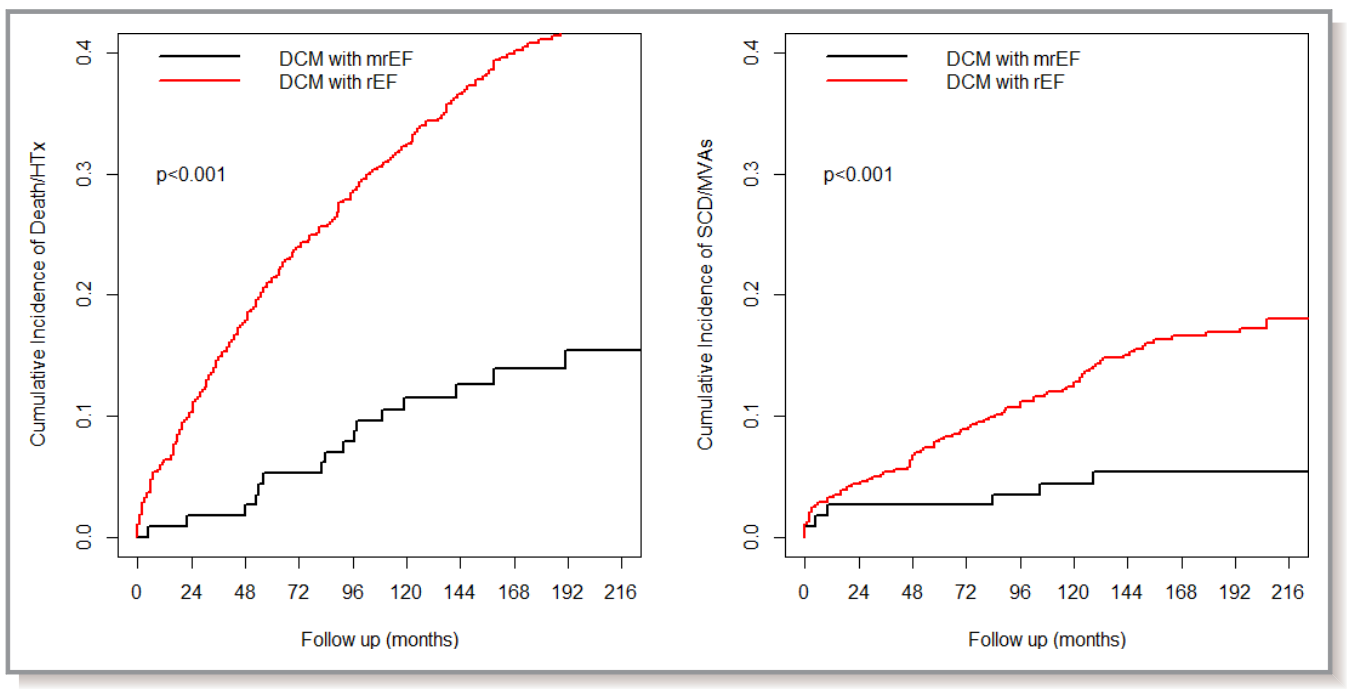

Figure 1. Cumulative incidence curves comparing dilated cardiomyopathy patients with mid-range ejection fraction and dilated cardiomyopathy patients with reduced ejection fraction in terms of death/ heart transplantation (left) and sudden cardiac death or major ventricular arrhythmias (right). At a median follow-up of 120 months, as compared with reduced ejection fraction patients, the mid-range ejection fraction group presented lower rates of all-cause death/heart transplantation (10\% vs $30 \%, P<0.001)$ and sudden cardiac death/major ventricular arrhythmias incidence (4\% vs $13 \%, P<0.001)$. DCM indicates dilated cardiomyopathy; HTx, heart transplantation; mrEF, mid-range ejection fraction; MVA, major ventricular arrhythmias; rEF, reduced ejection fraction; SCD, sudden cardiac death. 


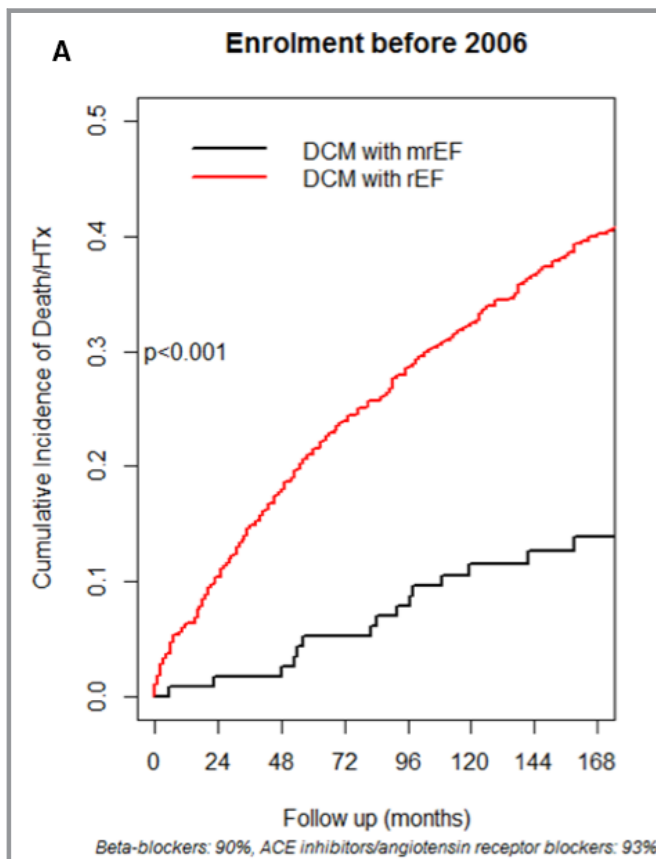

B

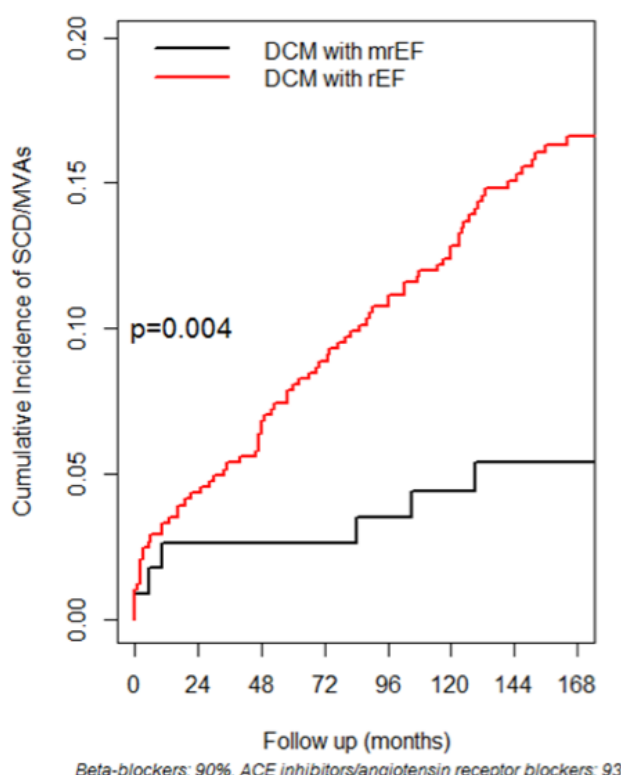

Enrolment after 2006

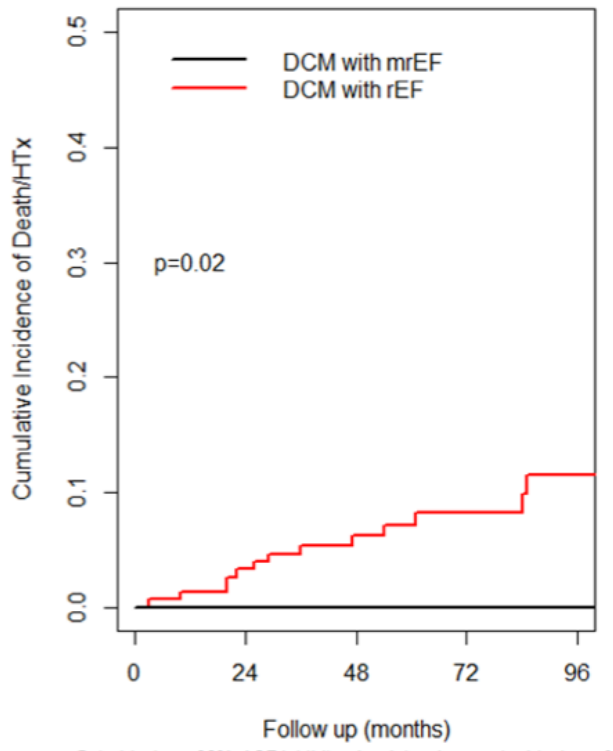

Beta-blockers: 93\%, ACE inhibitors/angiotensin receptor blockers: $94 \%$

\section{Enrolment after 2006}

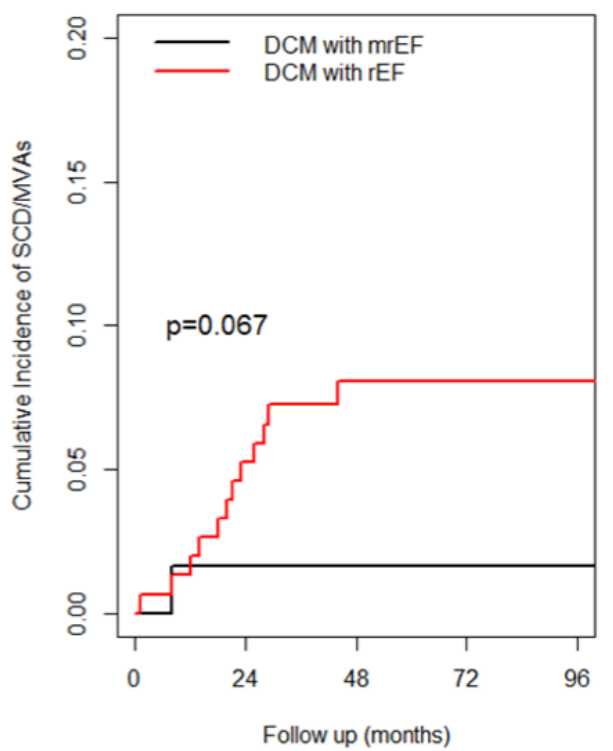

Beta-blockers: 93\%. ACE inhibitors/angiotensin receptor blockers: $94 \%$

Figure 2. Cumulative incidence curves comparing dilated cardiomyopathy patients with mid-range ejection fraction and dilated cardiomyopathy patients with reduced ejection fraction (rEF) in terms of death/heart transplantation (top panel, A) and sudden cardiac death or major ventricular arrhythmias (SCD/MVAs) (bottom panel, B), with stratification of the analysis by enrollment period. Lower incidences of events in terms of all-cause D/HTx and SCD/MVAs were reported in the DCM patients with mrEF regardless of the time of enrollment (before or after December 31, 2006). ACE indicates angiotensinconverting enzyme; DCM, dilated cardiomyopathy; HTx, heart transplantation; mrEF, mid-range ejection fraction; MVA, major ventricular arrhythmias; rEF, reduced ejection fraction; SCD, sudden cardiac death.

test as appropriate. For binary variables, the McNemar test was applied. Cumulative incidence curves for SCD/MVAs with respect to the competing risk of death for other causes or heart transplant were estimated and compared between groups. Cause-specific uni- and multivariable Cox regression models were estimated to find factors associated with $\mathrm{EF}$ 
worsening ( $E F<40 \%$ ), starting from the list of significant and clinically relevant parameters that emerged at univariable analyses and taking into account those with a $P$ value at univariable analysis of $<0.1$. Cumulative incidence curves for the risk of experiencing EF worsening with respect to the competing risk of death or heart transplant were estimated and compared between groups, stratified by the presence of the only significant risk factor derived from the previous analyses. A time-dependent Cox model was finally estimated to evaluate the impact of experiencing EF worsening on survival during follow-up, and an "extended" version of survival curves was estimated to visually represent results. ${ }^{18}$

\section{Results}

The study population included 812 patients with recently diagnosed DCM (median duration of symptoms of 2.5 [0-9] months). One hundred and seventy-five patients (22\%) presented with mrEF (ie, 40\%-49\%).

At baseline, in comparison with the $\mathrm{rEF}$ group, $\mathrm{mrEF}$ patients presented features of a less-advanced stage of disease, including smaller LV end-diastolic volume, lower rates of New York Heart Association functional classes III to IV, left bundle branch block, right ventricular dysfunction, moderate-severe MR, and restrictive LV filling pattern. No differences in the administration of angiotensin-converting enzyme inhibitors and beta-blockers were found; on the contrary, mrEF patients less frequently took diuretics and mineralocorticoid receptor antagonists (Table 1).

During a median follow-up time of 120 (60-204) months, 247 patients $(30 \%)$ died $(n=168)$ or underwent HTx $(n=79)$. One hundred and five patients (13\%) experienced an episode of SCD ( $n=59)$ or had MVAs $(n=46)$. At a median follow-up of 120 months, as compared with the rEF patients, the mrEF group presented lower rates of all-cause D/HTx (10\% versus $30 \%, P<0.001)$ and of SCD/MVAs incidence ( $4 \%$ versus $13 \%$, $P<0.001$ ) (Figure 1). A lower incidence of events in terms of all-cause D/HTX and SCD/MVAs was reported in mrEF patients than in rEF patients; this was true also upon stratifying the analysis by enrollment period (ie, before 2006 and from 2007, see Figure 2).

\section{Evolution in rEF}

Twenty-nine of $175 \mathrm{mrEF}$ patients (17\%) evolved to the $\mathrm{rEF}$ group at a median follow-up of 69 (24-91) months. Patients with worsened systolic function more frequently showed restrictive LV filling pattern at baseline in comparison with patients with persistent mrEF. Consequently, they were more frequently treated with diuretics and mineralocorticoid receptor antagonists (Table 2). In the time-dependent Cox
Table 2. Baseline Clinical Characteristics of the mrEF DCM Group Divided According to Evolution/No Evolution to rEF (LVEF $<40 \%)$

\begin{tabular}{|c|c|c|c|}
\hline & $\begin{array}{l}\text { No Evolution to } \\
\text { rEF Group } \\
(n=146)\end{array}$ & $\begin{array}{l}\text { Evolution to } \\
\text { rEF Group } \\
(n=29)\end{array}$ & $P$ Value \\
\hline Age, y & $42 \pm 6$ & $42 \pm 15$ & 0.84 \\
\hline Male sex (\%) & $91(62 \%)$ & $19(65 \%)$ & 0.74 \\
\hline NYHA III-IV, n (\%) & $6(4)$ & $3(10)$ & 0.15 \\
\hline $\mathrm{SBP}, \mathrm{mm} \mathrm{Hg}$ & $125 \pm 16$ & $129 \pm 18$ & 0.17 \\
\hline Heart rate, bpm & $69 \pm 12$ & $73 \pm 12$ & 0.14 \\
\hline LBBB (\%) & $29(19 \%)$ & $6(20 \%)$ & 0.94 \\
\hline \multicolumn{4}{|l|}{ Echocardiography } \\
\hline LAESAl, $\mathrm{cm}^{2} / \mathrm{m}^{2}$ & $9 \pm 2$ & $9 \pm 3$ & 0.82 \\
\hline LVEDDI, mm/m² & $31 \pm 3$ & $32 \pm 4$ & 0.72 \\
\hline LVEDVI, mL/m² & $70 \pm 18$ & $70 \pm 15$ & 0.67 \\
\hline LVEF, \% & $44.1 \pm 2.6$ & $44.3 \pm 3.0$ & 0.70 \\
\hline RVD, n (\%) & $12(8)$ & $5(17)$ & 0.15 \\
\hline $\begin{array}{l}\text { Moderate-severe } \\
\text { MR, } n(\%)\end{array}$ & $16(11)$ & $7(24)$ & 0.057 \\
\hline $\begin{array}{l}\text { Restrictive LV filling } \\
\text { pattern, } \mathrm{n}(\%)\end{array}$ & $4(2)$ & $4(14)$ & 0.02 \\
\hline $\mathrm{E} / \mathrm{E}^{\prime}$ & $10 \pm 3$ & $11 \pm 4$ & 0.11 \\
\hline sPAP & $24 \pm 5$ & $29 \pm 5$ & 0.017 \\
\hline \multicolumn{4}{|l|}{ Therapy } \\
\hline ACE-inhibitors-ARBs, n (\%) & $146(100)$ & $29(100)$ & 0.34 \\
\hline Beta-blockers, n (\%) & $127(88)$ & $26(88)$ & 0.82 \\
\hline Diuretics, n (\%) & $58(40)$ & $18(62)$ & 0.02 \\
\hline MRAs, n (\%) & $15(10)$ & $8(27)$ & 0.02 \\
\hline
\end{tabular}

Values are expressed as mean $\pm S D$ or median with interquartile range as appropriate, and as percentage. ACE indicates angiotensin-converting enzyme; ARBs, angiotensin receptor blockers; LAESAI, left atrial end systolic area indexed; LBBB, left bundle branch block; LV, left ventricular; LVEDDI, left ventricular end-diastolic diameter indexed; LVEDVI, left ventricular end-diastolic volume indexed; LVEF, left ventricular ejection fraction; MR, mitral regurgitation; MRAs, mineralocorticoid receptor antagonists; NYHA, New York Heart Association; RVD, right ventricular dysfunction; SBP, systolic blood pressure; SPAP, systolic pulmonary artery pressure.

model, DCM patients with persistent mrEF showed a significantly lower risk of long-term D/HTx than did those with baseline $\mathrm{rEF}$ (considering $\mathrm{rEF}$ as the reference category, hazard ratio: 0.08; $95 \% \mathrm{Cl}, 0.03-0.19$; Figure 3). Conversely, DCM patients with mrEF at baseline who, during follow-up, experienced a shift to $\mathrm{rEF}$ status presented a significantly higher risk of long-term D/HTx than did DCM patients with baseline rEF (hazard ratio: 2.27; 95\% Cl, 1.23-4.18; Figure 3). The presence of a restrictive LV filling pattern (hazard ratio: $4.0 ; 95 \% \mathrm{Cl}, 1.39-11.57, P=0.01$ ) emerged as the strongest baseline predictor independently associated with EF worsening after the stepwise selection starting from 


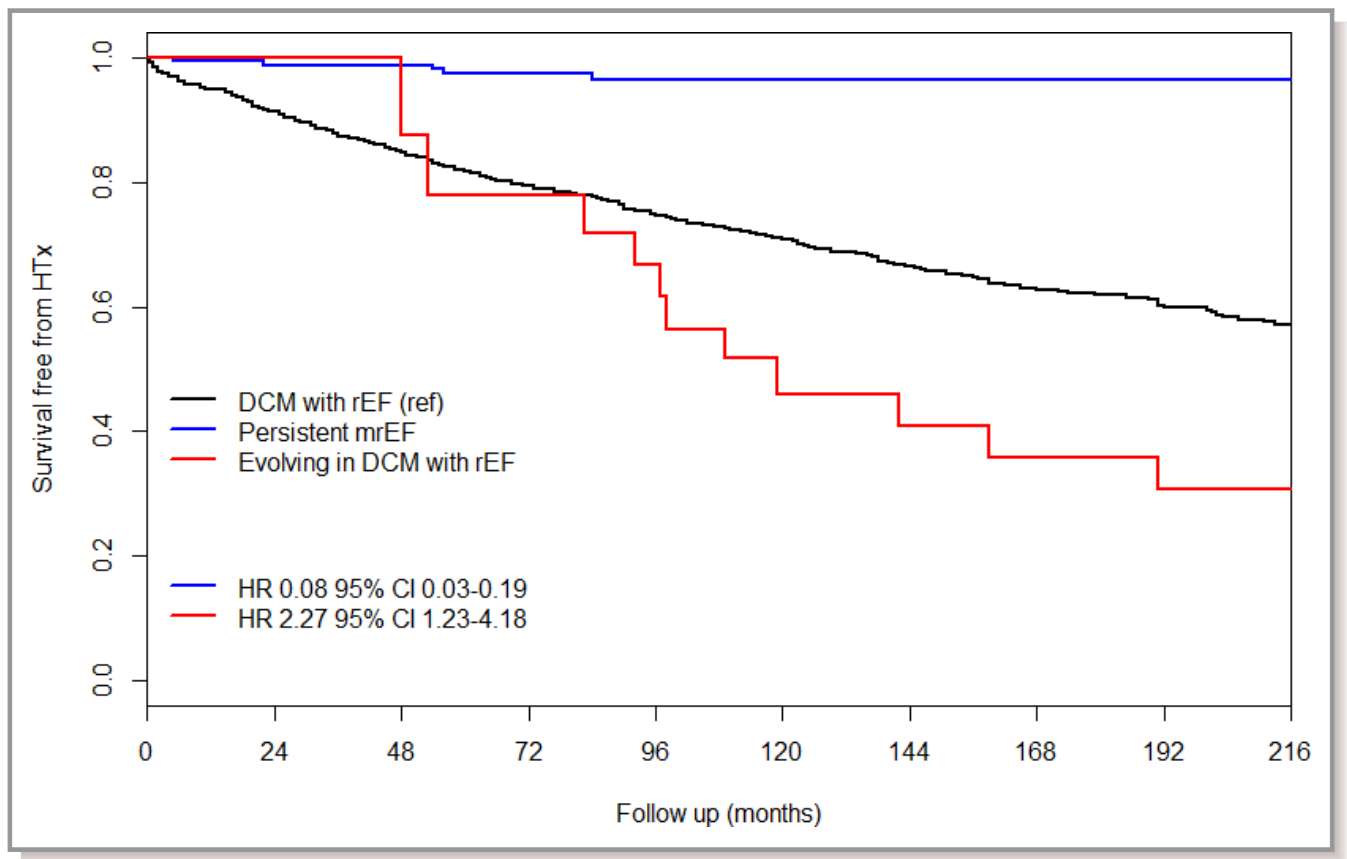

Figure 3. Extended Kaplan-Meier curves. Although dilated cardiomyopathy patients with mid-range ejection fraction at baseline globally exhibited a better long-term prognosis in comparison with dilated cardiomyopathy patients with reduced ejection fraction at baseline, the outcome of dilated cardiomyopathy patients with mid-range ejection fraction who experienced a worsening in their ejection fraction during follow-up progressively approached and became worse than that of dilated cardiomyopathy patients with reduced ejection fraction at baseline. DCM indicates dilated cardiomyopathy; HR, hazard ratio; HTX, heart transplantation; mrEF, mid-range ejection fraction; $r E F$, reduced ejection fraction

the list of significant parameters at univariable analyses (Table 3 and Figure 4).

\section{Discussion}

The existence of patients with mrEF is well-known in clinical practice. However, these patients have been included in clinical trials on preserved LVEF by using different EF cutoff values (eg, $>40 \%,>45 \%$, or $>50 \%$ ), regardless of ischemic or non-ischemic $\mathrm{HF}$ etiology. ${ }^{19-21}$ Following the introduction of the HFmrEF class in the last European Society of Cardiology guidelines, the need for a deeper understanding of this specific subgroup has been suggested. Nevertheless, recent data reported on HFmrEF often refer to evaluations in different moments of underlying disease course. ${ }^{22}$ Therefore, the real impact of $\mathrm{mrEF}$ at the time of diagnosis of $\mathrm{HF}$ is still lacking. Here, we described the natural history of mrEF from the time of disease presentation in a recently diagnosed DCM population. This is a strength of the present study, because limited information is available at this time in the literature on this subject. DCM patients represent a particular subgroup of the HF population. In fact, they are frequently asymptomatic or mildly symptomatic for $\mathrm{HF}$ at the time of diagnosis, often despite severe LV remodeling. ${ }^{2}$ Asymptomatic DCM patients deserve a careful and reliable determination of risk, because they could suffer rapid disease progression or die suddenly early after disease identification. ${ }^{5,8}$ This study provides a deep insight into the characterization and natural history of a large and well-selected cohort of DCM patients presenting with mrEF.

\section{Incidence and Long-Term Outcome of DCM Patients With mrEF}

Our findings show that almost one-quarter of DCM patients (22\%) had mrEF at baseline, with most of them (95\%) presenting with New York Heart Association functional classes I or II. Similarly, in the CHARM (Candesartan in Heart Failure: Assessment of Reduction in Mortality and Morbidity) trial, a consistent quota was found: 1295 of 7955 (17\%) HF patients had an LVEF between $43 \%$ and $52 \%{ }^{21}$ Thus, about one-fifth of newly diagnosed HF patients could present with $\mathrm{mrEF}$, and they should not be neglected.

The main clinical and echocardiographic characteristics at baseline found in this study indicate that the largest portion of mrEF DCM patients are diagnosed in a less-advanced stage of disease. An early diagnosis is probably supported by family screening programs or results from diagnostic tests performed in subjects being screened for sports participation. ${ }^{6}$ The resulting early optimal medical treatment probably has 
Table 3. Univariable Cause-Specific Cox Regression Analysis for EF Worsening

\begin{tabular}{|l|l|l|l|}
\hline \multirow{2}{*}{} & \multicolumn{3}{|l|}{ Unadjusted HRs } \\
\cline { 2 - 4 } & HR & $95 \% \mathrm{Cl}$ & $P$ Value \\
\hline Age, y & 1.009 & 0.981 to 1.037 & 0.525 \\
\hline SPB & 1.010 & 0.988 to 1.032 & 0.368 \\
\hline NYHA III-IV & 2.374 & 0.716 to 7.873 & 0.158 \\
\hline LBBB & 1.172 & 0.477 to 2.880 & 0.730 \\
\hline LAESAl & 1.041 & 0.913 to 1.186 & 0.551 \\
\hline LVEDVI & 0.995 & 0.976 to 1.013 & 0.563 \\
\hline LVEF & 1.031 & 0.899 to 1.182 & 0.660 \\
\hline Moderate-Severe MR & 2.346 & 1.001 to 5.498 & 0.050 \\
\hline Restrictive LV & $4.814^{*}$ & 1.662 to $13.947^{*}$ & $0.004^{*}$ \\
\hline filling pattern* & & & \\
\hline SPAP & 1.191 & 1.047 to 1.354 & 0.008 \\
\hline RVD & 2.234 & 0.848 to 5.887 & 0.104 \\
\hline LAESAl & 1.032 & 0.900 to 1.184 & 0.650 \\
\hline
\end{tabular}

HR indicates hazard ratio; LAESAI, left atrial end-systolic area indexed; LBBB, left bundle branch block; LV, left ventricular; LVEDVI, left ventricular end-diastolic volume indexed; LVEF, left ventricular ejection fraction; MR, mitral regurgitation; NYHA, New York Heart Association; RVD, right ventricular dysfunction; SBP, systolic blood pressure; sPAP, systolic pulmonary artery pressure.

*Stepwise Cox regression: starting from a list composed by moderate-severe MR, sPAP, and a restrictive LV filling pattern; this latter remained the only significant parameter, with adjusted HR: 2.896; $95 \% \mathrm{Cl}, 1.726-15.216 ; P=0.003$ (see Methods section, Statistical analysis subsection).

led to a significantly better prognosis in mrEF patients in comparison with rEF ones. Nevertheless, the benign prognosis of mrEF DCM patients is not generalizable to all cases. Importantly, a consistent percentage (ie, 17\%) of mrEF DCM patients evolved towards rEF at a median time of 5 years. There are a subgroup of DCM patients in which mrEF at presentation represented a transition zone in the way towards the rEF group. These data highlight the importance of an adequate early comprehensive characterization and of individualized long-term follow-up with recommended treatments performed in all DCM patients, including those apparently belonging to low-risk classes. In fact, in previous observational studies, renin-angiotensin antagonists were associated with greater benefits in patients with LVEF values of $40 \%$ to $49 \%$ in comparison with those with values of $\geq 50 \% .{ }^{23}$ In the TOPCAT trial (Treatment of Preserved Cardiac Function Heart Failure With an Aldosterone Antagonist), patients with EF values of $44 \%$ to $50 \%$ showed greater benefits when using spironolactone as compared with those with EF values of $>50 \%$. ${ }^{21}$ Accordingly, our results expand this information to application in a DCM subset and encourage clinicians to treat these patients with full HF therapies despite the absence of HF symptoms at enrollment, especially in the presence of diastolic dysfunction.

\section{The Prognostic Role of Diastolic Dysfunction}

Our analysis underlines the prognostic role of diastolic dysfunction in apparently low-risk DCM patients. At first evaluation, the presence of severe diastolic dysfunction (ie, restrictive LV filling pattern), associated with non-severe systolic dysfunction, emerged in our cohort as the strongest predictor of subsequent EF worsening (Figure 4). Importantly, patients with mrEF evolving to rEF presented a significantly higher risk of long-term major events as compared with baseline rEF patients. As previously reported in patients with non-dilated hypokinetic cardiomyopathy, the presence of significant diastolic dysfunction brought to light a subgroup with a high risk of adverse outcomes. ${ }^{8,10}$ This similar behavior could suggest a possible overlap between mrEF and non-dilated hypokinetic cardiomyopathy. A comprehensive and systematic genetic characterization, in association with a stricter follow-up regimen, may be necessary to better characterize these patients. In fact, a malignant genotype could be expressed without severe LV remodeling, such as mutations in LMNA A/C. ${ }^{22}$ Moreover, a "restrictive" phenotype has been included in the clinical spectrum of desmin-related cardiomyopathies. $^{23}$

\section{Limitations}

Our study population, partially referred to a tertiary center, along with the retrospective nature of the analysis, may have

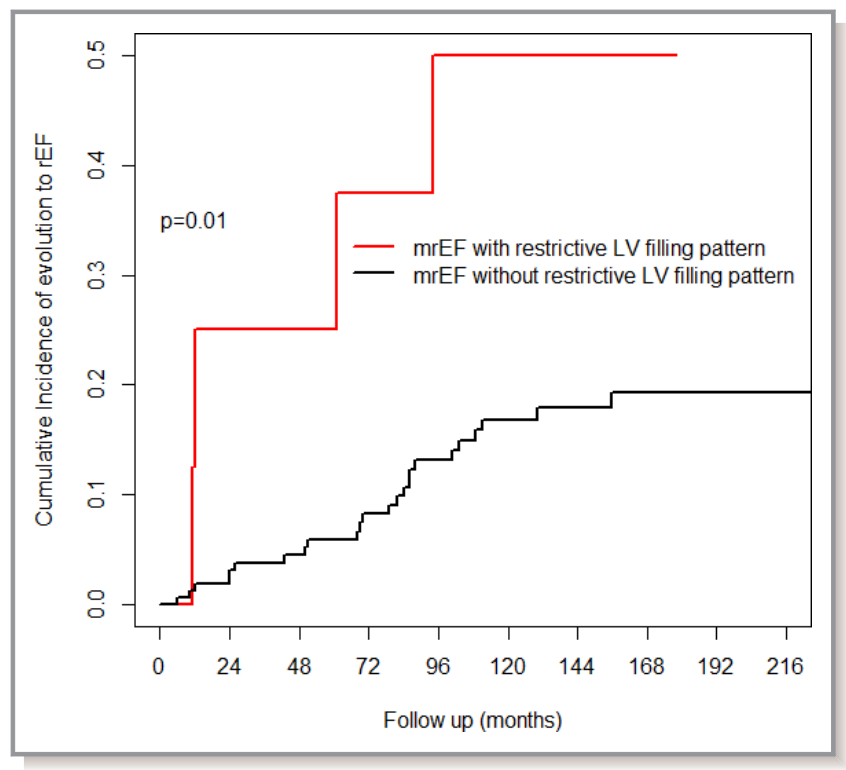

Figure 4. Cumulative incidence curves for ejection fraction worsening of mid-range ejection fraction dilated cardiomyopathy patients with or without a restrictive left ventricular filling pattern. The presence of a restrictive left ventricular filling pattern emerged as the strongest baseline predictor independently associated with ejection fraction worsening. LV indicates left ventricular; mrEF, midrange ejection fraction; $r E F$, reduced ejection fraction. 
created a degree of selection bias. Our results are applicable only to DCM patients and should not be generalized to the whole population of patients with HF. Although the study population represents, to the best of our knowledge, one of the largest existing mrEF DCM populations evaluated at baseline and during follow-up with a complete assessment, the low number of patients evolving to rEF underpowers the statistical analysis with only three potential predictors in the multivariable model. Dosages of medical HF therapy were not systematically available. However, according to the internal protocol, all patients uptitrated the therapy to the maximum tolerated dosage.

Systematic data on natriuretic peptides, 3-dimensional echocardiography, strain and strain rate, cardiac magnetic resonance, and genetic characterization findings were not available. They should be the focus of future larger studies.

\section{Conclusions}

mrEF denotes a consistent (ie, 22\%) subgroup of DCM patients who are generally diagnosed in an earlier stage and who present an apparently better clinical evolution. However, some mrEF DCM patients evolve into rEF despite medical therapy, with a subsequently worse prognosis. The presence of a restrictive filling pattern might be an important clue for identifying high-risk DCM patients presenting with mrEF.

\section{Disclosures}

None.

\section{References}

1. Ponikowski P, Voors AA, Anker SD, Bueno H, Cleland JGF, Coats AJS, Falk V, González-Juanatey JR, Harjola V-P, Jankowska EA, Jessup M, Linde C, Nihoyannopoulos P, Parissis JT, Pieske B, Riley JP, Rosano GMC, Ruilope LM, Ruschitzka F, Rutten FH, van der Meer P. 2016 ESC guidelines for the diagnosis and treatment of acute and chronic heart failure. Eur Heart $J$. 2016;37:2129-2200.

2. Merlo M, Cannatà A, Gobbo M, Stolfo D, Elliott PM, Sinagra G. Evolving concepts in dilated cardiomyopathy. Eur J Heart Fail. 2018;20:228-239.

3. McNally EM, Mestroni L. Dilated cardiomyopathy: genetic determinants and mechanisms. Circ Res. 2017;121:731-748.

4. Seidman JG, Seidman C. The genetic basis for cardiomyopathy: from mutation identification to mechanistic paradigms. Cell. 2001;104:557-567.

5. Merlo M, Pivetta A, Pinamonti B, Stolfo D, Zecchin M, Barbati G, Di Lenarda A, Sinagra G. Long-term prognostic impact of therapeutic strategies in patients with idiopathic dilated cardiomyopathy: changing mortality over the last 30 years. Eur J Heart Fail. 2014;16:317-324.

6. Moretti M, Merlo M, Barbati G, Di Lenarda A, Brun F, Pinamonti B, Gregori D, Mestroni L, Sinagra G. Prognostic impact of familial screening in dilated cardiomyopathy. Eur J Heart Fail. 2010;12:922-927.

7. Tsuji K, Sakata Y, Nochioka K, Miura M, Yamauchi T, Onose T, Abe R, Oikawa T, Kasahara S, Sato M, Shiroto T, Takahashi J, Miyata S, Shimokawa H. Characterization of heart failure patients with mid-range left ventricular ejection fraction-a report from the CHART-2 Study. Eur J Heart Fail. 2017;19:1258-1269.

8. Pinto YM, Elliott PM, Arbustini E, Adler Y, Anastasakis A, Bohm M, Duboc D, Gimeno J, de Groote P, Imazio M, Heymans S, Klingel K, Komajda M, Limongelli G, Linhart A, Mogensen J, Moon J, Pieper PG, Seferovic PM, Schueler S,
Zamorano JL, Caforio ALP, Charron P. Proposal for a revised definition of dilated cardiomyopathy, hypokinetic non-dilated cardiomyopathy, and its implications for clinical practice: a position statement of the ESC working group on myocardial and pericardial diseases. Eur Heart J. 2016;37:1850-1858.

9. Elliott P, Andersson B, Arbustini E, Bilinska Z, Cecchi F, Charron P, Dubourg O, Kuhl U, Maisch B, McKenna WJ, Monserrat L, Pankuweit S, Rapezzi C, Seferovic P, Tavazzi L, Keren A. Classification of the cardiomyopathies: a position statement from the European Society of Cardiology Working Group on Myocardial and Pericardial Diseases. Eur Heart J. 2008;29:270-276.

10. Gigli M, Stolfo D, Merlo M, Barbati G, Ramani F, Brun F, Pinamonti B, Sinagra G. Insights into mildly dilated cardiomyopathy: temporal evolution and longterm prognosis. Eur J Heart Fail. 2017;19:531-539.

11. Merlo M, Ammirati E, Gentile P, Artico J, Cannata A, Finocchiaro G, Barbati G, Sormani P, Varrenti M, Perkan A, Fabris E, Aleksova A, Bussani R, Petrella D, Cipriani M, Raineri C, Frigerio M, Sinagra G. Persistent left ventricular dysfunction after acute lymphocytic myocarditis: frequency and predictors. PLoS One. 2019;14:e0214616.

12. Spezzacatene A, Sinagra G, Merlo M, Barbati G, Graw SL, Brun F, Slavov D, Di Lenarda A, Salcedo EE, Towbin JA, Saffitz JE, Marcus FI, Zareba W, Taylor MR, Mestroni L; Familial Cardiomyopathy Registry. Arrhythmogenic phenotype in dilated cardiomyopathy: natural history and predictors of life-threatening arrhythmias. J Am Heart Assoc. 2015;4:e002149. DOI: 10.1161/JAHA.115. 002149.

13. Lang RM, Badano LP, Mor-Avi V, Afilalo J, Armstrong A, Ernande L, Flachskampf FA, Foster E, Goldstein SA, Kuznetsova T, Lancellotti P, Muraru $\mathrm{D}$, Picard MH, Rietzschel ER, Rudski L, Spencer KT, Tsang W, Voigt JU. Recommendations for cardiac chamber quantification by echocardiography in adults: an update from the American Society of Echocardiography and the European Association of Cardiovascular Imaging. I Am Soc Echocardiogr. 2015;28:1-39.e14.

14. Pinamonti B, Zecchin M, Di Lenarda A, Gregori D, Sinagra G, Camerini F. Persistence of restrictive left ventricular filling pattern in dilated cardiomyopathy: an ominous prognostic sign. J Am Coll Cardiol. 1997;29:604-612.

15. Rudski LG, Lai WW, Afilalo J, Hua L, Handschumacher MD, Chandrasekaran K, Solomon SD, Louie EK, Schiller NB. Guidelines for the echocardiographic assessment of the right heart in adults: a report from the American Society of Echocardiography endorsed by the European Association of Echocardiography, a registered branch of the European Society of Cardiology, and the Canadian Society of Echocardiography. J Am Soc Echocardiogr. 2010;23:685-688.

16. Zoghbi WA, Adams D, Bonow RO, Enriquez-Sarano M, Foster E, Grayburn PA, Hahn RT, Han Y, Hung J, Lang RM, Little SH, Shah DJ, Shernan S, Thavendiranathan P, Thomas JD, Weissman NJ. Recommendations for noninvasive evaluation of native valvular regurgitation: a report from the American Society of Echocardiography developed in collaboration with the Society for Cardiovascular Magnetic Resonance. I Am Soc Echocardiogr. 2017;30:303-371.

17. Stolfo D, Merlo M, Pinamonti B, Poli S, Gigli M, Barbati G, Fabris E, Di Lenarda A, Sinagra G. Early improvement of functional mitral regurgitation in patients with idiopathic dilated cardiomyopathy. Am J Cardiol. 2015;115:1137-1143.

18. Snapinn SM, Jiang Q, Iglewicz B. Illustrating the impact of a time-varying covariate with an extended Kaplan-Meier estimator. Am Stat. 2005;59:301307.

19. Yusuf S, Pfeffer MA, Swedberg K, Granger CB, Held P, McMurray JJV, Michelson EL, Olofsson B, Ostergren J. Effects of candesartan in patients with chronic heart failure and preserved left-ventricular ejection fraction: the CHARM-Preserved Trial. Lancet. 2003;362:777-781.

20. Pitt B, Pfeffer MA, Assmann SF, Boineau R, Anand IS, Claggett B, Clausell N, Desai AS, Diaz R, Fleg JL, Gordeev I, Harty B, Heitner JF, Kenwood CT, Lewis EF, O'Meara E, Probstfield JL, Shaburishvili T, Shah SJ, Solomon SD, Sweitzer NK, Yang S, McKinlay SM. Spironolactone for heart failure with preserved ejection fraction. N Engl J Med. 2014;370:1383-1392.

21. Solomon SD, Zile M, Pieske B, Voors A, Shah A, Kraigher-Krainer E, Shi V, Bransford T, Takeuchi M, Gong J, Lefkowitz M, Packer M, McMurray JJ; Prospective comparison of ARNI with ARB on Management Of heart failUre with preserved ejectioN fracTion (PARAMOUNT) Investigators. The angiotensin receptor neprilysin inhibitor LCZ696 in heart failure with preserved ejection fraction: a phase 2 double-blind randomised controlled trial. Lancet. 2012;380:1387-1395.

22. Chioncel O, Lainscak M, Seferovic PM, Anker SD, Crespo-Leiro MG, Harjola VP, Parissis J, Laroche C, Piepoli MF, Fonseca C, Mebazaa A, Lund L, Ambrosio GA, Coats AJ, Ferrari R, Ruschitzka F, Maggioni AP, Filippatos G. Epidemiology and one-year outcomes in patients with chronic heart failure and preserved, mid-range and reduced ejection fraction: an analysis of the ESC Heart Failure Long-Term Registry. Eur J Heart Fail. 2017;19:1574-1585.

23. Lh L, Benson L, Dahlström U, Edner M. Association between use of reninangiotensin system antagonists and mortality in patients with heart failure and preserved ejection fraction. JAMA. 2012;308:2108-2117. 